\title{
The role of retrospectively perceived parenting style and adult attachment behaviour in music performance anxiety
}

\author{
Anna Wiedemann ${ }^{1,2}$, Daniel Vogel ${ }^{2}$, Catharina Voss ${ }^{3}$, Manfred Nusseck $^{4}$ and Jana Hoyer ${ }^{3}$ \\ ${ }^{1}$ School of Psychology, University of Aberdeen, United Kingdom \\ ${ }^{2}$ Institute for Complex Systems and Mathematical Biology, University of Aberdeen, United Kingdom \\ ${ }^{3}$ Institute of Clinical Psychology and Psychotherapy, Technical University Dresden, Germany \\ ${ }^{4}$ Freiburg Institute for Musician's Medicine, University of Music Freiburg, Medical Center-University \\ of Freiburg, Faculty of Medicine, University of Freiburg, Germany
}

\section{Corresponding author:}

Anna Wiedemann, School of Psychology, University of Aberdeen, William Guild Building, Kings College, Old Aberdeen AB24 3FX, United Kingdom. Email: anna.wiedemann.15@aberdeen.ac.uk

Accepted for publication in Psychology of Music (online first: January 9, 2019). This paper is not the copy of the record and may not exactly replicate the authoritative document published in the journal. Please do not copy or cite without the author's permission.

The article can be found online: https://journals.sagepub.com/doi/full/10.1177/0305735618817877 


\begin{abstract}
This study aimed to examine the extent to which music performance anxiety relates to retrospectively perceived parenting style and adult attachment behaviour. Participants were 82 music students $\left(M_{\text {age }}=\right.$ 23.5 years, $S D=3.4)$ with the majority being vocal $(30.5 \%)$, string $(24.4 \%)$ or piano $(19.5 \%)$ students each with about 20 performance opportunities per year. Music performance anxiety was assessed using the German version of the Kenny Music Performance Anxiety Inventory. Parenting style was measured by retrospective self-report using the German version of the Measure of Parenting Style, adult attachment behaviour by the Relationship Questionnaire based on Bartholomew's four category model. Furthermore, general anxiety-related symptoms were assessed by the Severity Measure for Generalized Anxiety Disorder. Canonical and partial canonical correlation analyses were used to measure the dependence between multi-dimensional constructs: Both parenting style and adult attachment behaviour were related to music performance anxiety as measured by a performance-related sub-scale of the Kenny Music Performance Anxiety Inventory ( $r=0.45, p=0.01$ and $r=0.37, p=0.02$, respectively). The partial canonical correlation analysis, however, showed no significant relations between music performance anxiety and parenting styles or adult attachment behaviour. A strong link of music performance anxiety and generalised anxiety was found in all analyses. This study expands on more theoretical research in this area and provides first empirical insight into this complex multi-dimensional relationship.
\end{abstract}

Keywords: Music performance anxiety, parenting style, adult attachment behaviour, generalised anxiety, musicians 


\section{Introduction}

Although music performance anxiety (MPA) is as old as performance itself, the interest in its definition and prevalence, its causes and consequences and methods of therapeutic prevention and intervention only started a few decades ago. Debilitating performance anxiety is accompanied by a decrease in the quality of performance, as the affected person often struggles with unrealistic expectations of failure, as well as self-devaluation (Kenny, 2011). Theoretical approaches to understanding MPA are mainly based on other areas of psychological functioning considering psychodynamic and behavioural theories (Kenny, 2011). MPA is considered a special form of emotional behaviour involving a variety of aspects including motor expressive behaviour (e.g., trembling or shaky voice, hands, arms or legs), reactions of the autonomous nervous system (e.g., rapid heart rate, shortness of breath, loss of sensitivity or numbness), subjective feelings (e.g., feeling tense, helpless or inadequate), cognitive appraisal (e.g., concentration or memory problems) or behavioural changes (e.g., insomnia or avoidance reactions; Kesselring, 2006).

The most widely used scale to assess MPA is the Kenny Music Performance Anxiety Inventory (K-MPAI), which rests upon Barlow's emotion-based theory of anxiety disorders (Barlow, 1998; Kenny, 2009). The theory considers the origins of anxiety and related emotional behaviours to be an interacting set of three vulnerabilities (Barlow, 1998, 2000): generalised biological vulnerabilities, generalised psychological vulnerabilities and specific psychological vulnerabilities. Generalised biological vulnerabilities are the genetic contribution(s) to the expression of traits such as anxiety, neuroticism, negative affect and behavioural inhibition, which is estimated to run in a range of $30 \%$ to $50 \%$ of variance (Chang, Chang, Fang, Chang, \& Huang, 2017; Fisher DiLalla, Kagan, \& Reznick, 1994; Shimada-Sugimoto, Otowa, \& Hettema, 2015). Generalised psychological vulnerabilities are early-life experiences that shape the development of a sense of control (Nowicki \& Strickland, 1973). A positive parent-child relationship is one of the most significant determinants of healthy cognitive, emotional and social development and plays a key role in the development of self-concept and selfworth (Hesari \& Hejazi, 2011). Adverse early-life events, particularly an overcontrolling environment, may produce a sense of uncontrollability that may contribute to an increased negative affect as well as a higher risk of anxious or depressive behaviour (Barlow, 2000). Specific psychological vulnerabilities are the early learning experiences that may become relevant in the predisposition of individuals to focus anxiety on specific objects or events (Barlow, 1998, 2000. These specific psychological vulnerabilities are closely linked to generalised biological and psychological vulnerabilities (Barlow, 2000).

Although MPA is a complex construct of intra-psychic, intra-personal and social factors, research mainly focuses on psychological vulnerabilities to gain a further understanding of its causes and consequences, particularly to develop effective methods of therapeutic intervention for musicians where MPA becomes debilitating. As most adult musicians begin their musical education before the age of 12, and nearly half of these before the age of seven (Nagel, 1987), it seems plausible to assume early 
experiences may influence how musicians process, cope with and evaluate the extensive physical arousal and psychological demands of public performances. Findings based on other areas of psychological functioning show that individuals experiencing adverse parenting are more likely to display insecure attachment behaviours (Karavasilis, Doyle, \& Markiewicz, 2003), and have a significant higher risk of suffering from mood fluctuations and depressive or anxious behaviour (Oakley-Browne, Joyce, Wells, Bushnell, \& Hornblow, 1995; Yap \& Jorm, 2015). Hence, parenting and attachment are often discussed in relation to MPA (Kenny, 2011). Interestingly, previous research examining self-reported selfidentified causes of MPA showed it is believed that parenting behaviour only plays a minor role (Krawehl \& Altenmüller, 2000). Although the relationship of parenting or attachment behaviour with anxiety in general has been extensively studied (Bögels \& Brechman-Toussaint, 2006; McLeod, Wood, \& Weisz, 2007; Murray, Creswell, \& Cooper, 2009), research has rarely been expanded to more specific anxiety types such as MPA. Despite the theoretical discussion in literature, to our knowledge, no empirical evidence exists on how parenting or adult attachment behaviour may relate to MPA. On these grounds, it is the aim of this study to provide such empirical insights and expand on more general research in this area.

\section{Methods}

\subsection{Participants}

The sample consisted of 82 music students aged $18-33$ years $(M=23.5, S D=3.4 ; 69.5 \%$ women). An a priori power analysis based on Fisher's z transformation showed a sample size of $n=82$ can be seen as sufficient to draw statistically legitimate conclusions. Most participants were vocal, string or piano students. Other studied instruments included woodwind, plucked, percussion or brass as well as conducting (see Table 1). Most participants were either single ( $n=59,72.0 \%)$ or married/cohabiting with a partner $(n=21,25.6 \%)$ and rated their status of general health as excellent ( $n=19,23.2 \%)$, very good $(n=39,47.6 \%)$ or good $(n=22,26.8 \%)$. In total, 16 participants $(19.5 \%)$ indicated a regular medication intake such as hormonal contraception, antiallergic, or anti-depressant medication. Overall, 12 participants $(14.6 \%)$ reported a chronic illness.

\section{[Table 1]}

\subsection{Study design and procedure}

Data were provided by administering an online survey using LimeSurvey (Version 2.06+ Build 150731). All measurements were self-rating instruments. Participants were recruited nationwide via university mailing lists as well as locally at the University of Music in Dresden and Freiburg through face-to-face introductions in undergraduate lectures and seminars displaying survey invitations. Participants needed to be enrolled in a music programme (performance, pedagogy or music education) at an undergraduate 
or postgraduate level and show sufficient language proficiency in German to participate in the study. Study participation was entirely voluntary and could be withdrawn at any time without consequences. This study obtained ethical approval by the Ethics Committee of the Technical University Dresden (EK 28022012).

\subsection{Measures}

General information was obtained about age, sex, nationality, marital status, studied instrument/ time and specialisation, the number of performance opportunities per year, general state of health measured by the general health item of the Short-Form 36 (Ware \& Sherbourne, 1992), as well as regular use of medication and chronic diseases. Further assessment methods are outlined below.

\subsubsection{Assessing MPA}

MPA was measured using the German version of the Kenny Music Performance Anxiety Inventory (KMPAI; Kenny et al., 2004; Kenny, 2009) translated by Spahn, Walther and Nusseck (2016). The KMPAI includes 40 questions related to psychological vulnerability (nine items), negative cognitions (six items), proximal somatic anxiety (seven items), parental empathy (three items), memory (two items), pre- and post-performance rumination (two items), generational transmission of anxiety (three items), self/other scrutiny (three items), controllability (two items), opportunity cost (one item), trust (one item), and pervasive anxiety (one item). Participants rated each item on a seven-point Likert scale ranging from strongly disagree to strongly agree (possible sum score: 0-240 with higher scores indicating a stronger MPA). The K-MPAI shows high internal consistency (Cronbach's $\alpha=0.94$; Kenny et al., 2004). Given that the K-MPAI includes items that assume an underlying relationship of MPA with parenting style (e.g., item 9 "My parents were mostly responsive to my needs"), adult attachment behaviour (e.g., item 8 "I find it difficult to depend on others"), or general anxious disposition (e.g., item 19 "Sometimes I feel anxious for no particular reason"), a performance-related sub-scale was constructed in addition to the full version. This 24-item version (K-MPAI-24; possible sum score: 0-144) only included those items that were directly related to the performance situation and were reviewed by the first two authors independently (see Online Supplementary Material 1). The K-MPAI-24 showed similar internal consistency compared to the full version of the inventory (Cronbach's $\alpha=0.94)$.

\subsubsection{Assessing parenting style}

Parenting style was measured using the German version of the Measure of Parenting Style (MOPS; Parker et al., 1997), which is an abbreviated version of the Parental Bonding Index (Parker, Tupling, \& Brown, 1979). The MOPS represents an internationally accepted self-assessment instrument investigating the basic dimensions of retrospectively perceived indifference, abuse and overcontrol across 15 items in total. Participants were asked to choose the most suitable attitudes and behaviours of their parents before the age of 16 on a four-point Likert scale ranging from not true at all to extremely 
true. Higher scores of the MOPS indicate severity of the three domains (possible range for indifference: $0-18$, abuse: $0-15$, overcontrol: $0-12$ ). Validation of the German translation revealed satisfactory internal consistency (Cronbach's $\alpha$ for items ranged from 0.20 to 0.94 ), predictive validity $(82.2 \%$ of psychiatric diagnosis could be described by identifying parental style) as well as support for a threefactor solution (Rumpold, Doering, Höfer, \& Schüßler, 2002).

\subsubsection{Assessing adult attachment behaviour}

Adult attachment behaviour was assessed using the German version of the Relationship Questionnaire (RQ; Doll, Mentz, \& Witte, 1995) based on the model of attachment theory by Bartholomew (Bartholomew, 1990; Bartolomew \& Horowitz, 1991). The questionnaire represents four attachment prototypes based on "concept of self" and "concept of others": those include secure (positive concept of self and others), dismissive (positive concept of self, negative concept of others), preoccupied (negative concept of self, positive concept of others), and anxious (negative concept of self and others) attachment behaviours. Participants were asked to rate four statements, each corresponding to one of the four prototypes, on a seven-point Likert scale ranging from strongly disagree to strongly agree. Previous research confirmed stable test-retest reliability $\left(r_{t t}=0.64\right.$ [secure], $r_{t t}=0.73$ [dismissive], $r_{t t}=0.67$ [preoccupied] and $\mathrm{r}_{\mathrm{tt}}=0.69$ [anxious]; timespan 6 weeks) and reasonable congruent and discriminant validity of the RQ (Asendorpf, Banse, Wilpers, \& Neye, 1997).

For correlational models, participants' raw score for each prototype was entered into the model. In further analysis an overall attachment category was assigned for each participant. Those four scores for secure, dismissive, preoccupied and anxious attachment behaviour were projected on two scales reflecting concept of self and concept of others. The assigned prototype category as seen in Figure 1 was created as follows: The RQ score for secure attachment was projected on to the diagonal ray pointing northwest from the origin, likewise, the RQ score for dismissive attachment on the diagonal ray pointing southwest, and so forth. The resulting four vectors pointing diagonally away from the origin were superimposed by taking their sum. Based on which quadrant the resulting sum-vector lies in, one of the four attachment behaviour style prototypes was attributed to each participant as a categorical variable. As several points coincided with each other, due to the rather coarse scale of 1-7 for each of the original scores, a small random noise (a normal variate with mean zero and variance 0.16 ) was added to each point in Figure 1. This adjustment ensures that, for example, all 35 points are visible in the upper left quadrant such that the visual impression reflects the actual numbers.

\subsubsection{Assessing generalised anxiety}

Generalised anxiety was measured using the Severity Measure for Generalized Anxiety Disorder (Beesdo-Baum et al., 2012; Lebeau et al., 2012), a dimensional measure of the Diagnostic and Statistical Manual of Mental Disorders (DSM-5, American Psychiatric Association, 2013). The Severity Measure for Generalized Anxiety Disorder (GAD) includes 10 items about thoughts, feelings and behaviours, 
often tied to concerns about family, health, finances, school, or work that occurred during the past 7 days. Participants rated each question on a five-point Likert scale ranging from never to all of the time. The total score ranges from 0 to 40, with higher scores indicating greater severity of GAD. The average total score, calculated by dividing the raw score by the number of items, provides guidance of the severity of the individual's generalised anxiety disorder indicating none $(\mathrm{GAD}=0)$, mild $(\mathrm{GAD}=1)$, moderate $(\mathrm{GAD}=2)$, severe $(\mathrm{GAD}=3)$, or extreme $(\mathrm{GAD}=4) \mathrm{GAD}$.

\subsection{Data analyses}

Statistical analyses were performed using R version 3.3.3 (R Core Team, 2017) as well as IBM $®$ SPSS $®$ Statistics for Windows version 24.0 (IBM, 2016). Canonical and partial canonical correlation analyses were used to measure the dependence between multi-dimensional constructs. To further examine the effect of parenting style on MPA, we performed a linear regression of the K-MPAI-24 on the six MOPS scores (indifference, abuse and overcontrol, each for mother and father). For participants who indicated growing up without a female $(n=1)$ or male $(n=3)$ parent/caregiver, the sample median of each parenting domain was used in multivariate analyses. The effect of adult attachment behaviour on MPA was further investigated by a one-way analysis of variance (ANOVA) with K-MPAI-24 as dependent and RQ category as factor variable. We applied an alpha level of 0.05 for all statistical tests.

\subsubsection{Canonical correlation analysis}

As several psychological constructs analysed in this study are expressed by several numerical variables, for example, perceived parenting style (MOPS) and attachment behaviour (RQ), canonical correlation analysis (CCA; Hotelling, 1936) was used to assess the degree of statistical dependence between two groups of numerical variables. Using CCA, each group of variables is combined into a single numerical variable, then Pearson's correlation coefficient from the two new variables (the "canonical" variables) is determined. Although CCA is closely related to linear regression, it is more general as it allows the comparison of groups of variables of arbitrary size. This generality is indeed needed for the present study, for example, when examining perceived parenting style (six MOPS scores) and attachment behaviour (four RQ scores). Moreover, CCA provides an undirected measure of statistical dependence. The CCA was carried out using the R-package CCP.

\subsubsection{Partial canonical correlation analysis}

With partial canonical correlation analysis (PCCA) we investigated the multivariate dependence structure between the groups of variables to determine how the relationship between MPA and perceived parenting style is mediated by adult attachment behaviour and generalised anxiety and vice versa. The partial correlation coefficient is Pearson's correlation coefficient of the residuals of both variables being regressed onto the mediators. A zero-partial correlation indicates "conditional independence", namely, the relationship between both variables is fully explained by the mediators, the mediation is full. We 
extended this approach to groups of variables by regressing all variables onto the mediators and performing a CCA on the corresponding residuals, which corresponds to a partial canonical correlation analysis (PCCA; Rao, 1969; Timm \& Carlson, 1976). A zero-canonical partial correlation coefficient indicates conditional independence between both groups of variables, conditional on the mediators.

\section{Results}

\subsection{Descriptive analyses}

On average, participants scored 99.6 ( $S D=40.6$, range: $0-240)$ on the full version of the K-MPAI (KMPAI-40) and 65.4 ( $S D=28.7$, range: $0-144)$ on the performance-related subscale (K-MPAI-24). The K-MPAI-40 strongly correlated with the K-MPAI-24, $r=0.95, p<0.001$. The mean score of the KMPAI-40 did not differ from Spahn et al. (2016); however, it was significantly higher than values reported by Kenny (2012; $n=373, M=83.7, S D=40.7$; two-tailed independent t-test: $t(453)=3.2, p<$ $0.05, d=0.39)$. Descriptive results for MPA, parenting style, attachment behaviour and generalised anxiety are shown in Table 2.

[Table 2]

The mean score for secure attachment $(M=5.0, S D=1.6$, range: $1-7)$ was higher than any other attachment prototype (see Table 2). Distribution of assigned attachment behaviour prototype is shown in Figure 1. Most participants were securely attached $(n=38,46.3 \%)$ followed by dismissive $(n=21$, $25.6 \%)$, preoccupied $(n=14,17.1 \%)$ and anxious $(n=9,11.0 \%)$ adult attachment.

[Figure 1]

Participants' average generalised anxiety raw score, measured by GAD, was 6.8 ( $S D=6.2$, range: 0 40). The average total score was 0.7 ( $S D=0.5$, range: 0-4). A correlation with Pearson's $r=0.56, p<$ 0.001 has been found between the K-MPAI-24 and GAD (see Figure 2). All participants reporting moderate or severe generalised anxiety symptoms reported above-average K-MPAI-24 scores. However, participants reporting no or mild generalised anxiety were distributed fairly uniformly along the K-MPAI-24 scale.

[Figure 2]

\subsection{Multi-dimensional analyses}

\subsubsection{Unconditional dependencies}

In a first step, we computed measures for the unconditional or marginal dependence between each of the two constructs, that is, without conditioning on any third variables or constructs that may act as mediators. For constructs expressed as a single variable, for example, MPA and generalised anxiety, we 
report Pearson's correlation coefficient, for constructs expressed by groups of variables, we report canonical correlation coefficients. All unconditional dependence measures are shown in Figure 3. Because the K-MPAI-40 includes items explicitly referring to perceived parenting style, attachment behaviour or general anxiety, we excluded any items not directly related to the performance situation. In the following, we focus on the K-MPAI-24 as we consider it more appropriate for the questions at hand. All numbers reported refer to this inventory. The respective results for the K-MPAI-40 instead of the K-MPAI-24 can be found in the Online Supplementary Material 2.

[Figure 3]

\subsubsection{Conditional dependencies}

In a second step, we computed measures of conditional dependence between each two of the constructs given the respective other two. Assuming multivariate normality, partial correlation provides a measure of conditional dependence for single-variable constructs and the partial canonical correlation for multidimensional constructs. All conditional dependencies between MPA (K-MPAI-24) and the other constructs are shown in Figure 4.

[Figure 4]

\subsubsection{The effect of parenting style on MPA}

We further examined in detail the effect of parenting style on MPA by performing a linear regression of K-MPAI-24 on the six MOPS variables (indifference, abuse and overcontrol, each for mother and father). Positive $\beta$-coefficients are related to a higher K-MPAI-24 score, negative ones to a lower KMPAI-24 score. Positive $\beta$-coefficients were observed for abuse and overcontrol by both parents. This can be seen in Figure 5. The direction of $\beta$-coefficients for retrospectively perceived indifference was different for mother and father. The absolute values of all $\beta$-coefficients for all three MOPS dimensions were larger for the mother compared to father. Exact values of $\beta$-coefficients, standard errors and $95 \%$ confidence intervals (95\% CIs) can be found in Online Supplementary Material 3.

[Figure 5]

In a further investigation, the K-MPAI-24 was regressed on the six MOPS scores separately for female and male participants. The absolute $\beta$-coefficients of mother scores were larger than the respective father scores in all three dimensions (indifference, abuse and overcontrol). This was the case both for female and for male participants. A comparison of all sex-specific $\beta$-coefficients of parenting dimensions is shown in Figure 6, for example, overcontrol by the mother in female participants showed a positive $\beta$ coefficient compared to a negative $\beta$-coefficients in male participants. However, no further significance testing is reported given the small number of male participants $(n=25)$. Furthermore, the large $95 \%$ CIs 
suggest no significant differences between parenting domains. Again, exact $\beta$-coefficients, standard errors and 95\% CIs can be found in Online Supplementary Material 3.

[Figure 6]

\subsubsection{The effect of adult attachment behaviour on MPA}

A one-way ANOVA showed a significant main effect of adult attachment behaviour on MPA for the four attachment prototypes, $F(3,78)=10.4, p<0.001, \eta^{2}=0.28$. The lowest K-MPAI-24 mean score was found in dismissive $(M=46.4, S D=19.7)$, followed by secure $(M=62.5, S D=26.3)$, and anxious $(M=$ 86.8, $S D=30.6)$ attached participants. Participants with a preoccupied attachment behaviour exhibited the highest K-MPAI-24 mean score $(M=87.8, S D=22.9)$, however, this was not ignificantly higher than anxious attached individuals.

Participants with a positive concept of self (secure and dismissive attachment behaviour; $M=$ $56.7, S D=25.2, n=56$ ) scored significantly lower on the K-MPAI-24 compared to participants with a negative concept of self (preoccupied and anxious attachment behaviour; $M=87.4, S D=25.5, n=23$ ) with $t(80)=-4.9, p<0.001, d=1.21$. Post-hoc analyses using Bonferroni adjusted alpha levels showed a significant difference between K-MPAI-24 mean scores of individuals with secure and preoccupied attachment behaviour $\left(M_{\mathrm{sec}}=62.5, S D=26.3 ; M_{\text {preocc }}=87.8, S D=22.9\right), t(50)=-3.2, p=0.01, d=1.03$, and marginally significant differences in secure and anxious attached participants $\left(M_{\mathrm{sec}}=62.5, S D=26.3\right.$; $\left.M_{\text {anx }}=86.8, S D=30.6\right), t(45)=-2.4, p=0.06, d=0.85$, as well as significant differences between dismissive and preoccupied $\left(M_{\mathrm{dism}}=46.4, S D=19.7 ; M_{\text {preocc }}=87.8, S D=22.9\right), t(33)=-5.7, \mathrm{p}<0.001, d=$ 1.94 , and dismissive and anxious participants $\left(M_{\mathrm{dism}}=46.4, S D=19.7 ; M_{\mathrm{anx}}=86.8, S D=30.6\right), t(28)=-$ $4.3, p=0.001, d=1.57$.

\section{Discussion}

\subsection{Summary of results}

The influence of parenting and early life experiences has been considered an important aspect in the development of MPA (Kenny, 2011). Individuals experiencing adverse parenting styles are more likely to develop insecure attachment behaviours, a poorer self-concept and a higher susceptibility to suffer from mood fluctuations and anxiety (Karavasilis et al., 2003; McClun \& Merrell, 1998; Oakley-Browne et al., 1995; Thompson, 2008; Yap \& Jorm, 2015). However, it is unknown if and how these findings are transferable to more specific anxiety types such as MPA. This study provides first empirical insight into the relationship of MPA and retrospectively perceived parenting style and adult attachment behaviour. The main results are as follows: 
1. There is a moderate dependence between MPA and parenting style.

2. However, conditional on attachment behaviour and generalised anxiety, the link between MPA and parenting style is less pronounced and not significant in our sample.

3. The maternal behaviour appears to have a stronger effect than paternal behaviour on an individual's music performance anxiety.

4. Among the four attachment behaviour prototypes, individuals with a positive concept of self (dismissive or secure attachment) display lower MPA whereas individuals with a negative concept of self (preoccupied or anxious attachment) display higher MPA.

5. MPA and generalised anxiety are strongly related. This relationship remains unchanged when controlling for parenting style and adult attachment behaviour.

Below we discuss the findings and potential explanations in detail and outline the implications for clinical practice as well as for future research.

\subsubsection{Parenting, attachment and MPA}

A clear link between parenting style and MPA can be seen in unconditional analyses with a canonical correlation of about 0.45 . The link is less prominent and non-significant when considering adult attachment behaviour and generalised anxiety as potential mediators. Hence, results suggest an underlying relationship between MPA and parenting; this does not, however, appear very strong. Having a closer look into the effects of the mother's and father's parenting styles on MPA, we found a stronger influence of the mother. The sample consisted of more female than male participants, and the question arises whether this is a disguised same-sex-parent effect, namely if the father had a greater influence on developing MPA for males, whereas the mother did for females. Carrying out analyses for both sexes separately shows a consistently larger effect of the mother in both female and male participants. The unstandardised $\beta$-coefficients for individual parenting domains (indifference, abuse, overcontrol) vary in size and sign amongst male and female participants; however, this should not be over-interpreted: They are not large in comparison to the standard errors. The overlapping 95\% CIs indicate no significant pairwise differences. Moreover, the multi-dimensional interaction of the domains is unclear and not captured by a simple linear regression. Future empirical research into how parenting style may affect the development of MPA in males and females differently requires a larger sample, particularly of male participants.

A clear link between adult attachment behaviour and MPA can be seen in unconditional analyses, becoming less prominent and non-significant in multi-dimensional models. However, further analyses show that secure and dismissive relative to anxious and preoccupied attachment are significantly related to lower MPA, suggesting a positive self-concept plays an important role in this relationship. The group means differ by over one standard deviation, suggesting a large effect of selfconcept on MPA. This is in line with previous related research on the role of self-esteem in MPA, which 
demonstrates low self-esteem is a significant predictor of debilitating MPA (Sinden, 1999). Although, the self-concept seems to play a central role in MPA, it does not mean the concept of others is less important. Indeed, it may contribute to MPA depending on the type of attachment. For instance, dismissive attached individuals may view relationships to others as unimportant and see themselves as self-sufficient, hence, displacing a negative concept of others. On the contrary, anxious attached individuals, who also display a negative concept of others, may seek high levels of intimacy, approval and responsiveness by others, which may produce higher levels of stress in performance situations. Hence, these concepts are substantially different and may contribute to MPA in a different way.

\subsubsection{MPA and generalised anxiety}

The strongest link in multi-dimensional models is found in MPA and generalised anxiety, which remains unchanged in conditional analyses. Most participants reported no or mild generalised anxiety-related symptoms. Amongst those, below and above-average MPA scores were present. In contrast, participants with more severe GAD reported above-average K-MPAI scores only. The emerging pattern of two distinct groups, on the one hand, those where MPA appears rather isolated, and, on the other hand, those where MPA is strongly related to GAD, is in line with previous findings (Kenny, 2011). Beyond that, previous research illustrates high co-morbidity rates of generalised anxiety with other psychological vulnerabilities, for example, with emotional dysregulation (Marganska, Gallagher, \& Miranda, 2013) or depression (Goldberg, 2008; Kendler, Gardner, Gatz, \& Pedersen, 2006). This may play a decisive role for treatment approaches of severe MPA. Given the small number of participants reporting moderate to severe GAD, however, the emerging pattern of these two distinct groups needs further investigation and replication.

\subsection{Study Limitations}

In this study, participants were recruited by online link distribution. Accordingly, questions arise about the sample's representativeness and generalisability of the results for musicians on the whole. We cannot rule out a participation bias: Participants experiencing more severe performance anxiety may show an increased willingness to take part in the study. However, it is possible to compare the distribution of psychological measures of other studies with distribution characteristics of our sample. This may provide a rough guideline on the representativeness of the present sample. For instance, having a closer look into attachment prototypes, it can be seen the majority of participants are securely attached followed by dismissive, preoccupied and anxious attachment behaviour. The proportion of securely attached participants is slightly lower and the proportion of dismissively attached participants slightly higher than expected (Bakermans-Kranenburg \& van IJzendoorn, 2009). However, bearing in mind different assessment methods and measures used in different studies (e.g., self-report versus interview), the general proportions of different attachment behaviours in this study cohort seem to be satisfactory. 
Moreover, it is worth mentioning the self-rated general health of the sample with the majority rating their general health as excellent or (very) good.

\subsection{Clinical implications and further research}

Although the study results suggest an underlying relation between MPA and parenting, this relationship does not appear very strong. On the contrary, individuals' concept of self seems to have a large effect on MPA. However, it is unclear how and to what extent results may have treatment implications. To date, cognitive-behavioural therapy (CBT) focusing on the "here and now" is showing success and robust evidence of effectiveness as a primary method of treatment for individuals suffering from severe MPA (Wilson \& Roland, 2002).

Further research should examine whether the emerging characteristics of two distinct groups of individuals with more severe MPA can be seen in a larger sample with more participants reporting stronger generalised anxiety and, if this is the case, whether they may benefit from different approaches during the treatment process. Furthermore, it should be investigated whether those reporting more severe MPA and a clear expression of generalised anxiety also report a more adverse parenting style compared to those with isolated MPA. Given the small number of participants showing both high MPA and high GAD, we did not run any further statistical testing. It seems plausible that individuals showing mild generalised anxiety but strong MPA may effectively benefit from CBT. However, those suffering from severe generalised anxiety and MPA may also display other psychological vulnerabilities and a more complex pathopsychological profile. In this case, individuals may benefit from a more comprehensive therapeutic approach, which may include revisiting early-life experiences. Pursuing this further is an important topic for future research towards effective treatments of MPA.

Finally, we have constructed a performance-related item version (K-MPAI-24) of the K-MPAI, which we found to be highly correlated with the full version $(r=0.95, \mathrm{p}<0.001)$. The full version of the K-MPAI contains several items explicitly referring to retrospectively perceived parenting style, attachment behaviour or generalised anxiety. Hence, it measures MPA by assuming the relationships we were examining in this study. We found the K-MPAI-24 and K-MPAI-40 not only to be strongly correlated, but also to yield qualitatively the same results in the analysis (see Online Supplementary Material 2), leading to the same conclusions. Together, our results raise the question of whether items measuring retrospectively perceived parenting style or attachment behaviour are indeed needed to assess MPA and whether an inventory measuring actual performance-related experiences only (and possibly general anxiety disposition) may be more efficient.

\subsection{Conclusion}

This study provides the first empirical insight into, and adds noteworthy evidence to, the relationship of MPA, parenting style and adult attachment behaviour. We found a moderate link between MPA and parenting. Conditioning on adult attachment behaviour and generalised anxiety, however, the link is less 
pronounced and not significant in our sample. This suggests the underlying relation is not very strong. Furthermore, our results show that a positive self-concept, displayed by secure and dismissive attached individuals, is significantly related to lower MPA. A negative self-concept, displayed by preoccupied and anxious attached, however, is significantly related to higher MPA. Beyond that, our results indicate a strong relationship of MPA with generalised anxiety. The question of what kind of implication these findings may have for treatment remains open for further research.

\section{Acknowledgements}

The authors wish to thank all participating students and administrative staff of the music universities for help distributing the link of the online questionnaires. Furthermore, the authors would like to express their very great appreciation to Professor Katja Beesdo-Baum for general support, to Birgit Maicher for programming the basic version of the questionnaires and to Professor Hans-Christian Jabusch for his prolific commitment during subject recruitment and discussions in the later stages of the project. Additionally, the first author would like to thank Doctor Jasna Martinovic for valuable support in the initial stages of the research project.

\section{Author contributions}

Each of the authors listed has made substantial contribution to the conception or the design of the work $(\mathrm{AW}, \mathrm{CV}, \mathrm{JH})$, the acquisition $(\mathrm{AW}, \mathrm{MN})$, analysis $(\mathrm{AW}, \mathrm{DV})$ or interpretation of results $(\mathrm{AW}, \mathrm{DV})$. AW and DV drafted the manuscript and all co-authors were involved in revising it critically for important intellectual content and consented to be accountable for all aspects of the work.

\section{Declaration of conflicting interest}

The authors declare that there is no conflict of interest.

\section{Funding}

The author(s) received no financial support for the research, authorship, and/or publication of this article. 


\section{References}

American Psychiatric Association, (2013). Diagnostic and statistical manual of mental disorders (5th ed.). Arlington, VA: American Psychiatric Publishing.

Asendorpf, J. B., Banse, R., Wilpers, S., \& Neye, F. J. (1997). Beziehungsspezifische Bindungsskalen für Erwachsene und ihre Validierung durch Netzwerk und Tagebuchverfahren. Diagnostica, 43(4), 289-313.

Bakermans-Kranenburg, M. J., \& van IJzendoorn, M. H. (2009). The first 10,000 adult attachment interviews: Distributions of adult attachment representations in clinical and non-clinical groups. Attachment \& Human Development, 11(3), 223-263 doi: 10.1080/14616730902814762.

Barlow, D. H. (1998). Anxiety and its disorders: The nature and treatment of anxiety and panic. New York, NY: Guildford Press.

Barlow, D. H. (2000). Unraveling the mysteries of anxiety and its disorders from the perspective of emotion theory. The American Psychologist, 55(11), 1247-1263.

Bartholomew, K. (1990). Avoidance of intimacy: An attachment perspective. Journal of Social and Personal Relationships, 7, 147-178.

Bartolomew, K., \& Horowitz, L. M. (1991). Attachment styles among young adults: A test of a four category model. Journal of Personality and Social Psychology, 61, 226-244.

Beesdo-Baum, K., Klotsche, J., Knappe, S., Craske, M. G., Lebeau, R. T., Hoyer, J., . . Wittchen, H. U. (2012). Psychometric properties of the dimensional anxiety scales for DSM-V in an unselected sample of German treatment seeking patients. Depression and Anxiety, 29(12), 1014-1024 doi: 10.1002/da.21994.

Bögels, S. M., \& Brechman-Toussaint, M. L. (2006). Family issues in child anxiety: Attachment, family functioning, parental rearing and beliefs. Clinical Psychology Review, 26(7), 834-856 doi: 10.1016/j.cpr.2005.08.001.

Chang, C. C., Chang, H. A., Fang, W. H., Chang, T. C., \& Huang, S. Y. (2017). Gender-specific association between serotonin transporter polymorphisms (5-HTTLPR and rs25531) and neuroticism, anxiety and depression in well-defined healthy Han Chinese. Journal of Affective Disorders, 207, 422-428 doi: S0165-0327(16)30826-6.

Doll, J., Mentz, M., \& Witte, E. (1995). Zur Theorie der vier Bindungsstile: Meßprobleme und Korrelate dreier integrierter Verhaltenssysteme. Zeitschrift Für Sozialpsychologie, 26(3), 148-159. 
Fisher DiLalla, L., Kagan, J., \& Reznick, J. S. (1994). Genetic etiology of behavioural inhibition among 2-year-old children. Infant Behavior and Development, 17(4), 405-412 doi: 10.1016/01636383(94)90032-9.

Goldberg, D. (2008). Towards DSM-V: The relationship between generalized anxiety disorder and major depressive episode. Psychological Medicine, 38(11), 1671-1675. doi: 10.1017/S003329170800295X.

Hesari, N. K. Z., \& Hejazi, E. (2011). The mediating role of self-esteem in the relationship between the authoritative parenting style and aggression. Procedia - Social and Behavioral Sciences, 30, 1724-1730 doi: 10.1016/j.sbspro.2011.10.333.

Hotelling, H. (1936). Relations between two sets of variates. Biometrika, 28(3), 321-377.

IBM (2016). IBM® SPSS® Statistics for windows (Version 24.0 ed.). Armonk, NY: IBM Corp.

Karavasilis, L., Doyle, A. B., \& Markiewicz, D. (2003). Associations between parenting style and attachment to mother in middle childhood and adolescence. International Journal of Behavioral Development, 27(2), 153-164 doi: 10.1080/0165025024400015.

Kendler, K. S., Gardner, C. O., Gatz, M., \& Pedersen, N. L. (2006). The sources of co-morbidity between major depression and generalized anxiety disorder in a Swedish national twin sample. Psychological Medicine, 37(3), 453-462 doi: 10.1017/S0033291706009135.

Kenny, D. T. (2004). Music performance anxiety: Is it the music, the performance or the anxiety? Music Forum, 10(4), 38-43.

Kenny, D. T. (2009). The factor structure of the revised kenny music performance anxiety inventory. In Aaron Williamon, Sharman Pretty, \& Ralph Buck (Eds.), Proceedings of the International Symposium on Performance Science 2009, (pp. 37-41). Utrecht: European Association of Conservatoires (AEC). ISBN 9789490306014.

Kenny, D. T. (2011). The psychology of Music Performance Anxiety. New York, NY: Oxford University Press.

Kenny, D. T., Davis, P., \& Oates, J. (2004). Music performance anxiety and occupational stress amongst opera chorus artists and their relationship with state and trait anxiety and perfectionism. Journal of Anxiety Disorders, 18(6), 757-777 doi: S0887618503000689.

Kesselring, J. (2006). Music performance anxiety. In E. Altenmüller, M. Wiesendanger \& J. Kesselring (Eds.), Music, motor control and the brain (pp. 309-318). Oxford, UK: Oxford University Press. doi: 10.1093/acprof:oso/9780199298723.001.0001 
Krawehl, I., \& Altenmüller, E. (2000). Lampenfieber unter musikstudenten: Häufigkeit, ausprägung und "heimliche theorien". Musikphysiologie und Musikermedizin, 7(4), 173-178.

Lebeau, R. T., Glenn, D. E., Hanover, L. N., Beesdo-Baum Katja, Wittchen Hans - Ulrich, \& Craske, M. G. (2012). A dimensional approach to measuring anxiety for DSM-5. International Journal of Methods in Psychiatric Research, 21(4), 258-272. doi: 10.1002/mpr.1369

Marganska, A., Gallagher, M., \& Miranda, R. (2013). Adult attachment, emotion dysregulation, and symptoms of depression and generalized anxiety disorder. American Journal of Orthopsychiatry, 83, 131-141 doi: 10.1111/ajop.12001.

McClun, L. A., \& Merrell, K. W. (1998). Relationship of perceived parenting styles, locus of control orientation, and self-concept among junior high age students. Psychology in Schools, 35(4), 381390.

McLeod, B. D., Wood, J. J., \& Weisz, J. R. (2007). Examining the association between parenting and childhood anxiety: A meta-analysis. Clinical Psychology Review, 27(2), 155-172 doi: 10.1016/j.cpr.2006.09.002.

Murray, L., Creswell, C., \& Cooper, P. J. (2009). The development of anxiety disorders in childhood: An integrative review. Psychological Medicine, 39(9), 1413-1423 doi: $10.1017 /$ S0033291709005157.

Nagel, J. J. (1987). An examination of commitment to careers in music: Implications for alienation from vocational choice. Dissertation Abstracts International, 48, 1154-1155.

Nowicki, S., \& Strickland, B. R. (1973). A locus of control scale for children. Journal of Consulting and Clinical Psychology, 40, 148-154 doi: 10.1037/h0033978.

Oakley-Browne, M. A., Joyce, P. R., Wells, J. E., Bushnell, J. A., \& Hornblow, A. R. (1995). Adverse parenting and other childhood experience as risk factors for depression in women aged 18-44 years. Journal of Affective Disorders, 34(1) doi: 10.1016/0165-0327(94)00099-U.

Parker, G., Roussos, J., Hadzi-Pavlovic, D., Mitchell, P., Wilhelm, K., \& Austin, M. (1997). The development of a refined measure of dysfunctional parenting and assessment of its relevance in patients with affective disorders. Psychological Medicine, 27, 1193-1203.

Parker, G., Tupling, H., \& Brown, L. B. (1979). A parental bonding instrument. British Journal of Medical Psychology, 52, 1-10.

R Core Team. (2017). R: A language and environment for statistical computing. Vienna, Austria: R Foundation for Statistical Computing. 
Rao, B. R. (1969). Partial canonical correlation. Trabajos De Estadistica Y De Investigacion Operativa, 20, 211-219.

Rumpold, G., Doering, S., Höfer, S., \& Schüßler, G. (2002). A German version of the measure of parenting style (MOPS). [Der Fragebogen dysfunktionaler elterlicher Beziehungsstile (FDEB)]. Zeitschrift Für Psychosomatische Medizin und Psychotherapie, 48(1), 59-74.

Shimada-Sugimoto, M., Otowa, T., \& Hettema, J. M. (2015). Genetics of anxiety disorders: Genetic epidemiological and molecular studies in humans. Psychiatry and Clinical Neurosciences, 69(7), 388-401 doi: 10.1111/pcn.12291.

Sinden, L. M. (1999). Music performance anxiety: Contributions of perfectionism, coping style, selfefficacy, and self-esteem. Dissertation Abstracts International Section A: Humanities and Social Sciences, 60(3-A)

Spahn, C., Walther, J., \& Nusseck, M. (2016). The effectiveness of a multimodal concept of audition training for music students in coping with music performance anxiety. Psychology of Music, 44(4), 893-909 doi: 10.1177/0305735615597484.

Thompson, R. A. (2008). Early attachment and later development: Familiar questions, new answers. In J. Cassidy \& P. R. Shaver (Eds.), Handbook of attachment: Theory, research, and clinical applications (pp. 348-365). New York: Guilford Press.

Timm, N. H., \& Carlson, J. E. (1976). Part and bipartial canonical correlation analysis. Psychometrika, 4l(2), 159-176.

Ware, J. E., \& Sherbourne, C. D. (1992). The MOS 36-item short-form health survey (SF-36): I. conceptual framework and item selection. Medical Care, 30(6), 473-483.

Wilson, G. D., \& Roland, D. (2002). Performance anxiety. In R. Parncutt \& G. McPherson (Eds.), The science \& psychology of music performance (pp. 47-61). Oxford, UK: Oxford University Press.

Yap, M. B. H., \& Jorm, A. F. (2015). Parental factors associated with childhood anxiety, depression, and internalizing problems: A systematic review and meta-analysis. Journal of Affective Disorders, 175, 424-440. doi: 10.1016/j.jad.2015.01.050. 
Table 1. Sample Characteristics $(N=82)$.

\begin{tabular}{lc}
\hline & $\mathrm{n}(\%)$ \\
\hline Specialisation & \\
Music Education & $17(20.7)$ \\
Music Pedagogy & $8(9.8)$ \\
Music Performance & $57(69.5)$ \\
Studied Instrument & \\
Conducting & $3(3.7)$ \\
Brass & $1(1.2)$ \\
Percussion & $2(2.4)$ \\
Piano & $16(19.5)$ \\
Plucked & $4(4.9)$ \\
Strings & $20(24.4)$ \\
Voice & $25(30.5)$ \\
Woodwind & $9(11.0)$ \\
Unknown & $2(2.4)$ \\
\hline & \\
Time instrument/ voice studied & $14.8(4.5)$ \\
Instrumentalists & $9.5(3.9)$ \\
Singers & Years (SD) \\
\hline & $20(2-100)$ \\
\hline Performance opportunities per year & $30(2-80)$ \\
Instrumentalists &
\end{tabular}

